\title{
Analysis of EMC Technology for Power System Automation Equipment
}

\author{
Zhang Yong $^{1}$, Xie Lianke ${ }^{1}$, Zhang Guoying ${ }^{1}$, Li Yong ${ }^{2}$, Gong Quanquan ${ }^{1}$ \\ ${ }^{1}$ State Grid Shandong Electric Power Research Institute, Shandong Jinan, 250002 \\ ${ }^{2}$ State Grid Shandong Electric Power Company, Shandong Jinan, 250001
}

Keywords: power system; automated equipment electromagnetic interference; EMC technology

\begin{abstract}
With the development of the power grid in recent years, a lot of advanced technology and equipment have been applied in the power system, which greatly improves the automation level of the power system. However, such electric and electrical equipment units, relay protection devices, control devices and communication technology are affected by electromagnetic interference in running when applied in the power system, thus affecting the stability of the power system. To solve this problem, the electromagnetic compatibility (EMC) technology has been gradually developed. This paper mainly analyzes the electromagnetic interference sources of the electric power system automation equipment as well as EMC design of shielding technology, grounding technology and filter technology and the specific application of EMC and its technology in the electric power system automation equipment.
\end{abstract}

\section{Introduction}

The power system consists of a lot of primary equipment and secondary equipment, which may produce electromagnetic interference to the power system due to their own or external factors in the course of operation, thus affecting normal running of the electrical equipment and thereby resulting in power outages and other electrical safety accidents. EMC technology is to ensure that during the running of the power system the electrical equipment will not produce electromagnetic interference affecting the operation of the power system. As a new technology, EMC technology is developed with the advancement of the electric power industry. It is very important to analyze the operation safety and stability of the automated equipment of the electric power system.

\section{EMC technology}

EMC technology is developed with the advancement of the power electronic technology, electronic equipment and the like. As all electronic technology and electronic equipment produce electromagnetic interference in the operation, EMC technology is put forward to solve the electromagnetic interference of the electronic technology and electronic equipment. With the gradual development of the power grid to automation and intelligence, many advanced electronic equipment and electric equipment units are used in the power system, and the electromagnetic interference in the operation of the power system is increasingly serious. Therefore, electromagnetic compatibility has become a hard warranty in the electronic product design, which is directly related to the quality of the electronic products. In the design process, electromagnetic compatibility designer can reduce electromagnetic interference and improve the reliability of electronic equipment by a proper design approach.

\section{Electromagnetic interference source of the electric power system automation equipment}

The electromagnetic interference source of the electric power system automation equipment is complicated, as there is primary equipment, secondary equipment and external electromagnetic radiation, as well as internal components of the electrical equipment, transmission channels and other parts producing electromagnetic interference, which will cause interference to the automated equipment of the electric power system. As the automated equipment of the electric power system 
carries a microcomputer system, which contains a large number of digital circuits and analog circuits, most of which are integrated circuit blocks, diodes and A/D conversion circuits. They are both interference sources and also parts sensitive to the interference source sensitive. Especially, CMPS and D/A are parts the most sensitive to the interference source. The interference source signal is manifested in differential mode and common mode in the micro-computer system, and enters the micro-computer system via the power system, the transmission channel and the electrostatic field inside the electronic components. In general, power cables and input/output lines of the microcomputer system can generate a big current circuit to produce a very strong electromagnetic induction.

\section{Design method of EMC technology}

EMC technology can effectively limit the interference source from the interference to the power system and the electronic equipment, thus effectively compatible with all electrical equipment. EMC technology commonly used for automated equipment of the electric power system includes grounding technology, isolation technology, filtering technology, shielding technology and the like.

\subsection{Grounding technology}

Grounding technology is to establish a small-resistance conduction circuit between a ground plane chosen from the power system and a selected point, with all electronic components in the system interconnected but at 0 potential, for which the signals in other circuits can be referred to. If there is no current passing through 0 potential, there will be no voltage drop and the current of the interference source will be led to the ground through the grounding equipment, thus reducing the power supply of interference power. The power system is grounded in China by single point grounding, multi-point ground and mixed network grounding. Single point grounding is to connect all grounding leads to the earth through a single point, which is generally applied to the interference signal with an electromagnetic frequency below $1 \mathrm{MHz}$; multi-point grounding means that the grounding leads are contacted through multiple points, which, contrary to the single point grounding, is mainly used for the interference signal with an electromagnetic frequency above $10 \mathrm{MHz}$. Mixed network grounding is mainly used for complex grounding circuits, which mainly suppresses the interference signal with an electromagnetic frequency in 1-10mhz. The grounding design of the power system should take into account the following factors, as far as possible to reduce the electromagnetic interference caused by self-induction and mutual-induction eddy current of the grounding wire. The grounding wire should be as short as possible in length to reduce impedance.

\subsection{Filtering technology}

The filtering technology can restrain the interference of electromagnetic wave by filter. Electromagnetic filter is a filter circuit composed of capacitor, inductance and resistor. The filter can remove the frequency at a particular frequency point or the frequencies beyond the frequency point to get a specific power signal. The filter can effectively solve the electromagnetic interference and inhibit the radio interference, as well as electromagnetic interference produced at the transmitter output end and the receiver input end to filter out the interference signals and make the equipment compatible. The filter works in two ways, one is to reflect the useless signal back to the signal source and the other is to consume the useless signal in the filter. If the interference source is suppressed by filtering, it is required to first know the spectrum of the interference source and the distribution of the interference source in the frequency band. To obtain the correct numerical value, it is necessary to use a professional interference instrument to detect and select a specific filter. Common filters include digital filters, analog filters, surface acoustic wave filters, media filters and active power filters. The digital filter converts the input signal into a certain output signal to change the signal spectrum; the analog filter is a transducer commonly used in the test system; the media filter is made of the ceramic material with high dielectric constants, low loss and small thermal expansion coefficients, which is composed of several long resonators in longitudinally parallel by 
multiple stages or in series, characterized by advantages such as good power resistance, narrow bandwidth and small loss; the active power filter is an electronics device that can dynamically restrain harmonics and compensate harmonics and reactive powers according to the change of frequency as an ideal harmonic compensation device.

\subsection{EM shielding technology}

During the operation of the power system, power cables, transformers and other power equipment will produce some interference magnetic fields, which may cause some interference to the power system. Electromagnetic shielding technology is applied by certain technical means to shield the electromagnetism generated by electrical equipment within a certain range, to reduce the interference generated by the magnetic field to the automated equipment of the electric power system. Common electromagnetic shielding methods include electric shielding, magnetic shielding and electromagnetic shielding. The electric shielding is mainly applied to shield the electrostatic field produced by the electric power system and the electrical equipment; the magnetic shielding is mainly applied to shield the magnetic field of the power system, thus shielding the electromagnetic interference. In the specific application, different electromagnetic compatibility technologies should be employed according to the installation locations and models of the automated equipment of the electric power system.

\subsection{Isolation technology}

During the operation of the power system, there is some interference magnetic field around the feeder. If there are other wires nearby, there will be some electromagnetic coupling action, which can cause some interference to the power system, so effective measures should be taken to isolate the interference signals and electromagnetic field in the operation of the power system. Isolation must be made in accordance with the following principles: first, the interference line cannot be arranged in parallel to other lines, if arrangement in parallel is required, the ratio of wire spacing to wire diameter should not be greater than 40 . Under normal circumstances, it is best to keep a larger spacing between the wires and the section in parallel should be as short as possible. If the general line is arranged in parallel to a sensitive line, the spacing between the lines must be greater than $50 \mathrm{~mm}$. Second, the power feeder should be isolated from the power signal line. If the two are in parallel, the spacing should not be greater than $50 \mathrm{~mm}$. Third, high frequency conductors must be isolated from other interference lines. Fourth, some pulse lines have a large power and cause a lot of signal interference to other lines, so the pulse lines should be regarded as interference lines.

\section{Application of electromagnetic compatibility technology in the power system automation equipment}

The power system automation equipment consists of microcomputer system, power circuit, peripheral drive circuit, communication circuit, peripheral circuit, A/D conversion circuit, D/A conversion circuit and other circuits. During the design and in the analysis of the electromagnetic compatibility of the power system automation equipment, it is also required to take into account the electromagnetic compatibility between the electrical equipment and other systems. At present, the electromagnetic compatibility technology of the power system automation equipment in China mainly involves the following techniques: the first is frequency design technique, which is a major difficulty in the design of the whole computer system as the microcomputer system has to use the uniform frequency in the design to guarantee the frequency characteristics. Frequency design involves level verification, design of the highest operating frequency, frequency reduction and harmonic separation, and other technologies. The second is grounding technology design, including two parts: power supply internal resistance analysis technology and design of ground contact and ground wire. The analysis of power supply internal resistance mainly focuses on the maximum instantaneous power of the supply, lest the grounding technology cannot meet the requirements of the maximum instantaneous power and thus affecting the whole power system. The system with weak power should be separated with that with strong power in the design of ground contact and 
ground wire. The third is wiring technology design. In the power system design, the impact of each connection wire and pin is reduced to isolate the electromagnetic interference signal. It is required to have an effective understanding of the distribution of all power system parameters in wiring. Wiring technology mainly involves layering technology, wire diameter selection and surrounded wiring. The fourth is the power technique, which is applied to reduce the effect of the electromagnetic interference. Power technology design requires taking into account two points: the design of power characteristics, such as current absorption capacity and power margin; selection of the power nature, such as batteries or rectifier batteries, distributed power supply or centralized power supply. The fifth is frequency decrease and control technique, which is applied to decrease the frequency of the output signal in the normal operation of the power system. For some large power output signals, the frequency should be decreased, for example, appropriate resistors or capacitors are added to the LED circuit to decrease the frequency of the output signal with large power. The sixth is surface veneering technique, which is a circuit making technology integrating the integrated circuits and printed circuit boards. As the integrated circuits are unprocessed bare chips in the factory, the circuit making technology can be applied to paste bare chips to the surface of the printed circuit boards. In this way, the circuit is small in size with excellent electromagnetic compatibility. The seventh is software technique. With the development of science and technology, there is increasing external interference to the power system. The interference of the external signal destroys normal programs of the power system automation equipment and there may be program interruption, no respond, chip program rewrite and other errors, thus causing malfunction or touch of the electrical equipment. Therefore, there is need to control the power equipment program by the software technology.

\section{Conclusion}

With the development of computer, information technology, artificial intelligence and control technology, the power system automation equipment gradually develops to be miniaturized, multi-functionalized and integrated, which puts forward higher requirements for the electromagnetism compatibility of the power system automation equipment. Therefore, it is of great significance for the development of the power system automation to strengthen the research on the electromagnetic compatibility technology of the power system automation equipment.

\section{References}

[1] Zhang Zhenfeng. EMC Technology for Power Automation Equipment [J]. Tech Entrepreneur, 2014, (4): 83-83.

[2] Zhang Rushan. EMC Technology for Power System Automation Equipment [J]. Technological Innovation and Application, 2013, (35): 293-293.

[3] Bao Chenguang. Discussion on Application of EMC Technology in Power System Automation Equipment [J]. Electronics World, 2017, (10): 138-138.

[4] Hou Lei. EMC Technology for Power System Automation Equipment [J]. Private Science and Technology, 2014, (12): 26-27.

[5] Yue Juan, Di Yulong. EMC Technology for Power System Automation Equipment [J]. Sciences \& Wealth, 2017, (36): 205-205.

[6] Zhang Changbin, Chen Hongwei, Zhang Xinqiang. EMC Technology for Power System Automation Equipment [J]. Dual Use Technologies \& Products, 2016, (22): 175-175.

[7] Guo Xiaomin. Discussion on Application of EMC Technology in Power System Automation Equipment [J]. Telecom Power Technologies, 2017, (4): 238-239.

[8] Gao Changbi. Analysis of EMC Technology for Power System Automation Equipment [J]. Computer Fans, 2017, (14):158-159. 